\title{
ORIGINAL RESEARCH \\ Regional Gray Matter Atrophy in Patients with Parkinson Disease and Freezing of Gait
}

A. Tessitore

M. Amboni

G. Cirillo

D. Corbo

M. Picillo

A. Russo

C. Vitale

G. Santangelo

R. Erro

M. Cirillo

F. Esposito

P. Barone

G. Tedeschi
BACKGROUND AND PURPOSE: FOG is a troublesome symptom of PD. Despite growing evidence suggesting that FOG in PD may be associated with cognitive dysfunction, the relationship between regional brain atrophy and FOG has been poorly investigated.

MATERIALS AND METHODS: Optimized VBM was applied to $3 T$ brain MR images of 24 patients with PD and $12 \mathrm{HC}$. Patients were classified as either FOG - or FOG $+(n=12)$ based on their responses to a validated FOG Questionnaire and clinical observation. All patients with PD also underwent a detailed neuropsychological evaluation.

RESULTS: The VBM analysis in patients with FOG + showed a reduced GM volume in the left cuneus, precuneus, lingual gyrus, and posterior cingulate cortex compared with both patients with FOG - and $\mathrm{HC}$. We did not detect any significant change of $\mathrm{GM}$ volume when comparing $\mathrm{HC}$ versus all patients with PD (FOG - and FOG +). FOG clinical severity was significantly correlated with GM loss in posterior cortical regions. Finally, patients with FOG + scored lower on tests of frontal lobe function.

CONCLUSIONS: Our findings provide the first evidence that the development of FOG in patients with $\mathrm{PD}$ is associated with posterior GM atrophy, which may play a role in the complex pathophysiology of this disabling symptom.

ABBREVIATIONS: ${ }^{18} \mathrm{~F}=$ fluorine $18 ; \mathrm{FOG}=$ freezing of gait; FOG-Q $=$ FOG Questionnaire; FWE $=$ family-wise error; $\mathrm{GM}=$ gray matter; $\mathrm{HC}=$ healthy controls; $\mathrm{PD}=$ Parkinson disease; $\mathrm{RCPM}=$ Raven's Colored Progressive Matrices; UPDRS = Unified Parkinson's Disease Rating Scale; $\mathrm{VBM}=$ voxel-based morphometry
$\mathbf{F}$ OG is a common and very disabling symptom of PD in which patients experience an episodic inability to generate effective stepping despite the intention to walk. ${ }^{1}$ Recent studies identified FOG as 1 of the most relevant contributing factors to the worsening quality of life among patients with $\mathrm{PD}{ }^{2}$ Although typically occurring late in the progression of $\mathrm{PD}$, FOG can be experienced in relatively earlier stages and has been reported in up to $26 \%$ of patients never treated with levadopa. ${ }^{3}$ FOG has been suggested as an independent cardinal sign of PD not correlated with rigidity or bradykinesia but associated with gait, balance, and speech dysfunctions. ${ }^{4}$ In the deprenyl and tocopherol antioxidative therapy of parkinsonism cohort, early occurrence of FOG was associated with cognitive decline, ${ }^{5}$ and recent neuropsychological evidence has demonstrated a relation between cognitive dysfunction and FOG in patients with $\mathrm{PD} .{ }^{6,7}$

The mechanisms underlying FOG are not yet fully understood. ${ }^{8}$ Nonconventional imaging studies have yet to investigate the neural correlates of FOG showing an involvement of

Received November 24, 2012; accepted after revision January 10, 2012.

From the Department of Neurological Sciences (A.T., G.C., A.R., M.C., G.T.), Second University of Naples, Naples, Italy; Department of Neurological Sciences (M.A., M.P., C.V., G.S., R.E., F.E., P.B.I, University "Federico II," Naples, Italy; Institute for Diagnosis and Care "Hermitage Capodimonte" (M.A., D.C., A.R., G.T.), Naples, Italy; Department of Medicine and Surgery (P.B.), University of Salerno, Salerno, Italy; University of Naples "Parthenope" (C.V.), Naples, Italy; and Neuropsychology Laboratory (G.S.), Department of Psychology, Second University of Naples, Caserta, Italy.

A. Tessitore and M. Amboni contributed equally contributed to the article.

Please address correspondence to Gioacchino Tedeschi, MD, Division of Neurology, University of Naples, SUN, Piazza Miraglia 2-I-80138, Naples, Italy; e-mail: tessitorealessandro@libero.it

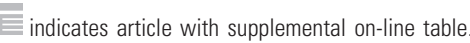

http://dx.doi.org/10.3174/ajnr.A3066 both posterior cortical ${ }^{9}$ and subcortical areas. ${ }^{10,11}$ However, to our knowledge, only 1 study ${ }^{10}$ has investigated the pattern of brain atrophy in patients with FOG, demonstrating reduced GM volume in the brain stem without differences in cortical areas between patients and HC. Hence, the aim of the present study was to further explore the differences in local GM volume in patients with PD with and without FOG by using VBM.

\section{Materials and Methods}

\section{Patient Population}

We investigated 24 patients ( 7 women and $17 \mathrm{men}$ ) with a diagnosis of PD according to the clinical diagnostic criteria of the UK Parkinson's Disease Society Brain Bank. ${ }^{12}$ Inclusion criteria were the following: 1) age of 45 years or older, 2) a Hoehn and Yahr stage $\leq 2.5$ while in "on medication state," 3) disease duration $<10$ years, and 4) antiparkinsonian treatment at a stable and optimized daily dosage during the 4 weeks before study entry. Exclusion criteria were the following: 1) dementia according to clinical diagnostic criteria for dementia associated with $\mathrm{PD}^{13} ; 2$ ) major depression according to the criteria of the Diagnostic and Statistical Manual of Mental Disorders-IV for current major depression ${ }^{14}$; 3) clinically significant comorbidities, including serious cardiovascular or cerebrovascular disease; and 4) anticholinergic or neuroleptic treatment. Patients were classified as exhibiting FOG (FOG + ) based on the following 2 conditions that both had all to be fulfilled: 1) score $>0$ to item 3 of the FOG-Q, ${ }^{15}$ and 2) patient recognition of this condition when it was demonstrated to them by an experienced clinician miming the phenomenon. Patients not fulfilling either of the above conditions were classified as FOG-. A group of 12 age- and sex-matched $\mathrm{HC}$ with no previous histories of neurologic or psychiatric diseases and with normal brain MRI findings was also recruited to assess FOG-related effects in relation to the 
healthy population. All participants gave written informed consent, which was approved by the local ethics committee according to the Declaration of Helsinki.

\section{Clinical, Motor, and Neuropsychological Assessment Analysis}

All patients were evaluated 60-90 minutes after their morning dose of levadopa and underwent a detailed clinical and cognitive evaluation.

We recorded demographic data and disease history, and administered parts I-IV of the UPDRS ${ }^{16}$ and the FOG-Q. ${ }^{15}$ To evaluate cognitive function, we administered the following: 1) Mini-Mental State Examination, 2) the spatial (Corsi Block-Tapping) and verbal span ${ }^{17}$ to test spatial and verbal memory, 3) Rey Auditory Verbal Learning Test (15-word) to assess immediate and delayed recall of word lists, 4) the constructional apraxia test, ${ }^{17} 5$ ) the RCPM test to evaluate abstract nonverbal reasoning, 6) Frontal Assessment Battery, a brief battery aimed at assessing frontal lobe functions, 7) phonological verbal fluency to estimate mental flexibility, 8) the Stroop Test (part II and III) to assess both color vision and sensitivity to interference, 9) Ten-Point Clock Test to evaluate spatial programming, and 10) cancellation attentional matrices ${ }^{17}$ to explore attention domain. Test scores were corrected for current normative values. Motor and cognitive functions were each evaluated by 2 different raters. Tests were administered by a trained neuropsychologist blinded to presence/absence of FOG.

\section{Imaging Parameters}

All subjects were scanned in the morning, and each patient was in the "on-medication" state (ie, 60-90 minutes after their first dose of levadopa). MR images were acquired on a $3 \mathrm{~T}$ scanner (GE Healthcare, Milwaukee, Wisconsin) equipped with an 8-channel parallel head coil. Structural MR imaging data were acquired by using 3D T1weighted sagittal images (Signa HDxt 3T twinspeed GE sequence inversion-recovery fast-spoiled gradient recalled, TR $=6988 \mathrm{~ms}$, $\mathrm{TI}=1100 \mathrm{~ms}, \mathrm{TE}=3.9 \mathrm{~ms}$, flip angle $=10^{\circ}$, voxel size $=1 \times 1 \times$ $1.2 \mathrm{~mm}^{3}$ ). Gross anatomic abnormalities were ruled out by an experienced neuroradiologist who was blind to clinical diagnosis and evaluated MR imaging scans for each subject.

\section{Statistical Analysis of Clinical, Motor, and Neuropsychological Data}

Demographic, clinical, and neuropsychological features of patients with FOG + and FOG - were compared by a $t$ test for independent samples or Fisher exact test, as appropriate.

Given the small number of subjects in each group and to avoid type 1 errors, we evaluated between-group comparisons on cognitive measures with the Mann-Whitney $U$ test, a nonparametric analysis. Computation was supported by the Statistical Package for the Social Sciences, Version 16 (SPSS, Chicago, Illinois) software. Significance threshold was set to $P \leq .05$.

\section{$V B M$}

Data were processed and examined by using SPM8 software (http:// www.fil.ion.ucl.ac.uk/spm), where we applied VBM implemented in the VBM8 toolbox (http://dbm.neuro.uni-jena.de/vbm.html) with default parameters incorporating the DARTEL toolbox in SPM8. This was used to obtain a high-dimensional normalization protocol. ${ }^{18}$ Images were bias-corrected, tissue-classified, and registered by using linear (12-parameter affine) and nonlinear transformations (warping) within a unified model. ${ }^{18,19}$ Subsequently, the warped GM seg- ments were affine-transformed into Montreal Neurological Institute space and were scaled by the Jacobian determinants of the deformations to account for the local compression and stretching that occurs as a consequence of the warping and affine transformation (modulated GM volumes).$^{20}$ Finally, the modulated volumes were smoothed with a Gaussian kernel of 8-mm full width at half maximum. The GM volume maps were statistically analyzed by using the general linear model based on Gaussian random field theory. Statistical analysis consisted of an analysis of covariance with total intracranial volume and age as covariates of no interest. Moreover, neuropsychological data differing between patients with and without FOG were entered as covariates of no interest in an additional analysis of covariance aimed at ruling out the possibility that GM differences were caused by these specific variables. For each group, we defined linear contrasts ( 1 as group of interest; -1 as a comparing group; 0 as group of noninterest) to test for differences in GM volumes between the groups: 1) each group versus control subjects (patients with FOG+ versus HC: [-1 01 1]; patients with FOG - versus HC: $\left.\left.\left[\begin{array}{lll}0 & -1 & 1\end{array}\right]\right) ; 2\right)$ all patient groups (FOG + and FOG - ) versus HC $[-1-11]$; and 3 ) each group versus the other (patients with FOG + versus patients with FOG$\left[\begin{array}{lll}-1 & 1 & 0\end{array}\right]$; FOG - patients versus patients with FOG $\left.+\left[\begin{array}{lll}1 & -1 & 0\end{array}\right]\right)$. Because we did not have an a priori hypothesis concerning a specific region of interest, we applied a whole-brain statistical threshold of $P \leq .05$, FWE-corrected for multiple comparisons. Only clusters comprising 100 or more voxels were reported.

To correlate GM volume changes and FOG-Q score, we performed a correlation analysis by using the multiple regression function of SPM8. Correlation analyses were performed inside and outside specific ROIs. We considered ROIs to be the identified regions that showed the most significant GM change in the comparisons between groups. The resulting region-of-interest image was transformed into a binary mask that was applied explicitly to compute regression analysis within $\mathrm{PD}$ groups by using a significance level of $P \leq .05$, FWE-corrected for multiple comparisons. For exploratory purposes, we subsequently performed correlation analyses on the whole-brain level outside the ROIs (using a statistical threshold of $P \leq .001$, uncorrected). The coordinates of voxels exhibiting the greatest group effects were transferred from Montreal Neurological Institute space to Talairach space by using M. Brett's transformation (http://www.mrc-cbu.cam.ac.uk/Imaging/contents.html).

\section{Results}

\section{Clinical Findings}

The demographic characteristics of the patients are shown in the On-line Table. Among 24 patients with PD, 12 were classified as FOG + and 12 patients, as FOG - . No significant differences were observed between patients with FOG + and FOG - in age and sex; disease duration; Hoehn and Yahr stage; UPDRS I, III, IV scores; symptom-dominant side; and dopaminergic treatment. As expected, the FOG-Q and UPDRS II scores were significantly higher in FOG + compared with patients with FOG-; in particular, the UPDRS II score differed significantly between patients with FOG + and those with FOG - due to the presence in the scale of 2 gait-related items (item 14: freezing; item 15: walking). The 2 groups did not differ on the tremor UPDRS subscore. The total postural instability gait disturbances UPDRS subscore significantly differed between the 2 groups. However, because freezing (item 14) is relevant for the postural instability gait disturbances 


\begin{tabular}{|c|c|c|c|c|}
\hline Cognitive Tasks & $\begin{array}{c}\mathrm{FOG}+(n=12) \\
(\text { mean } \pm \mathrm{SD})\end{array}$ & $\begin{array}{c}\mathrm{FOG}-(n=12) \\
(\text { mean } \pm \mathrm{SD})\end{array}$ & $U$ Value & $P$ Value \\
\hline$\overline{M M S E}$ & $28.27 \pm 2.22$ & $27.94 \pm 1.58$ & 67.00 & .799 \\
\hline Corsi Span & $4.27 \pm 0.64$ & $4.77 \pm 0.92$ & 44.00 & .314 \\
\hline Verbal Span & $3.54 \pm 0.66$ & $4.02 \pm 0.70$ & 45.50 & .211 \\
\hline Immediate recall (Rey) & $34.54 \pm 8.44$ & $42.64 \pm 9.30$ & 33.00 & $.044^{\mathrm{b}}$ \\
\hline Delayed recall (Rey) & $7.65 \pm 2.90$ & $9.34 \pm 2.73$ & 40.00 & .118 \\
\hline CA & $10.85 \pm 0.71$ & $10.95 \pm 1.77$ & 55.50 & .525 \\
\hline RCPM & $26.00 \pm 4.98$ & $27.4 \pm 3.21$ & 53.50 & .449 \\
\hline FAB & $12.41 \pm 1.74$ & $15.67 \pm 1.27$ & 3.00 & $.001^{\mathrm{b}}$ \\
\hline Verbal fluency & $28.52 \pm 7.95$ & $43.61 \pm 8.87$ & 16.00 & $.001^{\mathrm{b}}$ \\
\hline Stroop (part II) & $37.20 \pm 10.26$ & $35.87 \pm 10.21$ & 65.50 & .976 \\
\hline Stroop (part III) & $18.42 \pm 4.58$ & $22.38 \pm 5.71$ & 37.00 & .079 \\
\hline TPCT & $4.42 \pm 2.71$ & $7.67 \pm 2.67$ & 24.00 & $.005^{\mathrm{b}}$ \\
\hline Attentional matrices & $43.21 \pm 9.11$ & $48.36 \pm 5.44$ & 43.50 & .169 \\
\hline
\end{tabular}

Note:-CA indicates constructional apraxia; FAB, Frontal Assessment Battery; MMSE, Mini-Mental State Examination; TPCT, Ten-Point Clock Test

a Scores are age- and education-adjusted.

Significant.
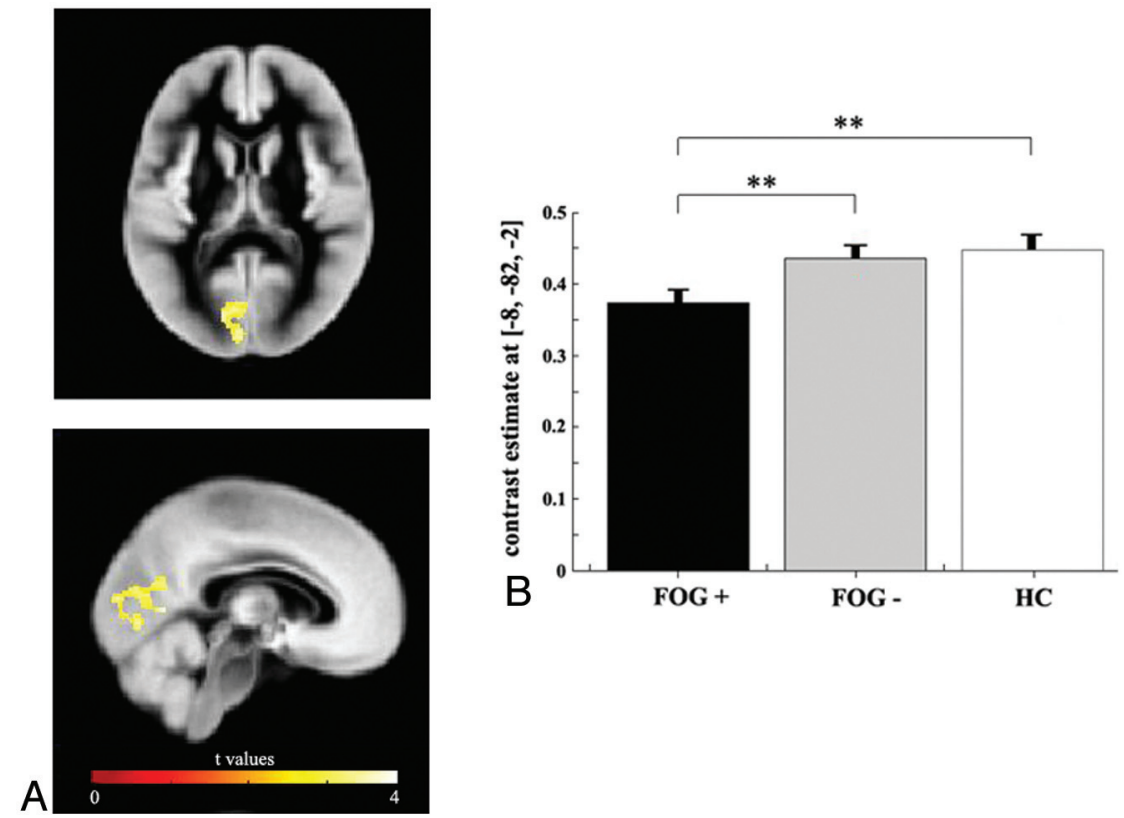

Fig 1. A, Areas of GM tissue loss deriving from the direct comparison between patients with FOG + and those with FOG - . The peak differences can be found within the left cuneus, precuneus, lingual gyrus, and posterior cingulate cortex. Results are superimposed on representative axial and coronal sections of the customized GM template, at a threshold of $P \leq .05$, FWE-corrected. B, Mean GM volume loss ( \pm standard error of the mean) extracted from the region (Statistical Parametric Mapping coordinates $\mathrm{x}:-8, \mathrm{y}$ : $-82, \mathrm{z}$ : -2 ) showing a maximal statistical difference between groups. Double asterisks indicate significance at the whole-brain statistical threshold corrected for multiple comparison, $(P \leq .05$, FWE-corrected).

subscore, the differences between the 2 groups disappeared when not considering the freezing score (our grouping variable) in the analysis (On-line Table).

\section{Neuropsychological Data}

Patients with FOG+ performed significantly worse than patients with FOG - on several cognitive tests. In particular, patients with FOG + compared with patients with FOGexhibited lower scores on the Frontal Assessment Battery, phonological verbal fluency, and Ten-Point Clock Test, with the first 2 exploring frontal-executive domains and the last mainly investigating spatial programming skill, which requires both executive and visuospatial functions. Furthermore, patients with FOG + exhibited reduced mean immediate recall of the Rey Auditory Verbal Learning Test 15-word score (Table 1).

\section{VBM Findings}

There were no differences in global GM, WM, or CSF volume between groups (patients versus HC; patients with FOG + versus patients with $\mathrm{FOG}^{-}$) (On-line Table). The analysis of regional volume differences revealed that patients with $\mathrm{PD}$ as a group (FOG + and FOG - ) showed no significant GM differences compared with $\mathrm{HC}$ at a threshold of $P \leq .05$, FWEcorrected. However, a significant difference in GM volume was detected when the FOG + and FOG - groups were compared. In particular, the FOG+ group showed reduced GM volume in a cluster including the left cuneus, precuneus, lingual gyrus, and posterior cingulate cortex compared with the FOG - group (Fig 1 and Table 2) $(P \leq .05$, FWE-corrected). Moreover, patients with FOG+ exhibited reduced GM volume in the left cuneus and lingual gyrus compared with HC (Table 2) ( $P \leq .05$, FWE-corrected), whereas, no differences in 


\begin{tabular}{|c|c|c|c|c|c|c|c|c|}
\hline \multirow[b]{2}{*}{ Contrast } & \multirow[b]{2}{*}{ Region } & \multirow[b]{2}{*}{ BA } & \multicolumn{3}{|c|}{ Talaraich Coordinates } & \multirow[b]{2}{*}{$\mathrm{T}$ Value } & \multirow[b]{2}{*}{ Cluster Size $\left(k_{E}\right)$} & \multirow[b]{2}{*}{$P_{\text {FWE-corr }}$} \\
\hline & & & $x$ & $Y$ & $Z$ & & & \\
\hline \multirow[t]{4}{*}{$\overline{\mathrm{FOG}}+<\mathrm{FOG}-$} & L posterior cingulate & 30 & -1 & -62 & 9 & 3.90 & 785 & $.025^{\mathrm{a}}$ \\
\hline & $\mathrm{L}$ ingual gyrus & 18 & -7 & -78 & 2 & 3.77 & & \\
\hline & $L$ cuneus & 18 & -12 & -78 & 14 & 3.54 & & \\
\hline & L precuneus & 23 & -4 & -61 & 20 & 3.40 & & \\
\hline \multirow{2}{*}{$\mathrm{FOG}+<\mathrm{HC}$} & L cuneus & 30 & -2 & -68 & 6 & 4.53 & 374 & $.015^{\mathrm{a}}$ \\
\hline & L ingual gyrus & 18 & -7 & -81 & 1 & 4.27 & & \\
\hline
\end{tabular}

Note:- $k_{E}$ indicates cluster size; FWE-corr, FWE-corrected; BA, Brodmann area; L, left a Significant.

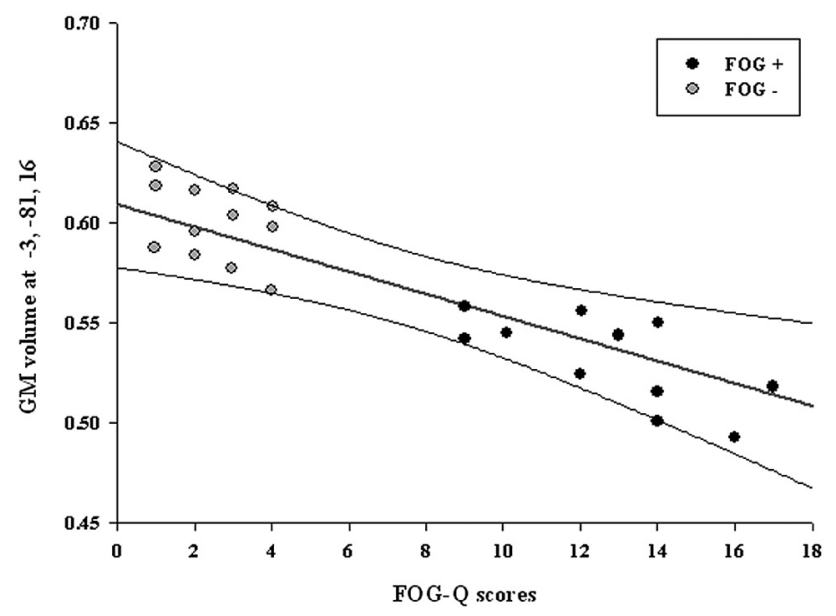

Fig 2. Negative correlation between the left cuneus GM volume and FOG- 0 scores in all patients with PD (FOG + and FOG-). Scatterplot shows distribution of the mean GM volumes (y-axis) of all voxels within the left cuneus cluster and FOG-0 scores (x-axis). The regression line (red) and the 95\% confidence intervals (black lines) are shown.

GM volume were identified in the comparison between $\mathrm{HC}$ and patients with FOG-. Moreover, we did not detect other significant GM differences between groups according to linear contrasts ( $\mathrm{HC}$ versus $\mathrm{PD}$ and $\mathrm{FOG}-$ versus FOG+) previously defined.

\section{Correlation Analyses}

Across all patients with $\mathrm{PD}$, a significant negative correlation was detected between FOG-Q scores and GM loss in the left occipital region (Statistical Parametric Mapping coordinates $\mathrm{x}:-3, \mathrm{y}:-81, \mathrm{z}: 16$; $t$ value: 6.43 ; $r$ value: -0.58 ; cluster $[\mathrm{k}]=$ 43; $P \leq .05$, FWE-corrected; Fig 2). No significant correlations were detected in voxels outside the ROIs.

\section{Discussion}

The aim of our study was to investigate structural brain differences between patients with PD with and without FOG. Our findings revealed that patients with FOG + exhibited left parietal (precuneus), occipital (cuneus and lingual gyrus), and posterior cingulate cortex atrophy compared with both patients with FOG - and patients with HC. Moreover, consistent with previous studies, we showed worse performance on several cognitive tests in patients with FOG + compared with patients with FOG-. Our imaging findings were not driven by the observed cognitive differences between patients with FOG + and patients with FOG - and a significant negative correlation was identified between GM loss of the left occipital cortex and FOG-Q scores.
Gait is traditionally seen as an automatic motor task that requires minimal higher cognitive input. However, a growing body of evidence has recently drawn attention to the importance of cognition in normal walking, which relies not only on the sensorimotor system but also on cerebral networks subserving attention, executive functions, and visuomotor integration. $^{21,22}$ Mental loading and divided attention may increase the occurrence of FOG episodes ${ }^{23}$; however, visual and auditory cues can usually overcome FOG. ${ }^{24}$ Furthermore, Helmich et $\mathrm{al}^{25}$ demonstrated an increased dependence on visual-information processing during a motor imagery task in $\mathrm{PD}$, suggesting that patients with $\mathrm{PD}$ increasingly rely on visual cues to control locomotion. ${ }^{26}$

Our findings are consistent with previous radiotracer studies demonstrating hypometabolism or hypoperfusion of temporoparietal and occipital cortices in patients with FOG. In particular, a $\left[{ }^{18} \mathrm{~F}\right]$-dihydroxyphenylalanine and FDG-PET study comparing patients with PD with and without FOG revealed that those with FOG had both reduced $\left[{ }^{18} \mathrm{~F}\right]$-dihydroxyphenylalanine uptake in the caudate and posterior putamen and reduced FDG uptake in posterior parietal areas. ${ }^{27}$ Moreover, hypometabolism of parietal, temporal, and occipital sensory association cortices was found to limit the effect of deep brain stimulation of the subthalamic nucleus in patients with FOG with PD, suggesting that the metabolic activity of these areas could contribute to the emergence of this disabling symptom. ${ }^{28}$ A recent fMRI study during imagined walking ${ }^{10}$ has shown less activation in the superior parietal lobule and anterior cingulate cortex of patients with PD compared with $\mathrm{HC}$. When the patients were divided into FOG + and FOGgroups, only patients with FOG + showed decreased activation in the frontal and posterior parietal cortices. This pattern has been confirmed in another recent study. ${ }^{29}$

Consistent with previous neuropsychological evidence, ${ }^{6,30}$ patients with FOG+ performed worse than those with FOG - on several cognitive tests. In particular, patients with FOG + displayed predominant executive-visuospatial impairment as evidenced by reduced scores on the Frontal Assessment Battery, phonemic verbal fluency, and Ten-Point Clock Test. These data suggest a prominent dysfunction of the dorsolateral prefrontal cortex, which has wide connections with posterior cortical areas, namely the parietal and occipital cortices. ${ }^{31}$ In the occipital cortex, the "ventral stream" (occipitotemporal pathway) is crucial for the identification of objects, whereas the "dorsal stream" (occipitoparietal pathway), which consists of reciprocal projections to the prefrontal and premotor cortices, is involved in mapping the spatial 
relationships of objects, thereby supporting visually guided movements. ${ }^{32,33}$

Our cognitive and neuroimaging findings are in agreement with previous VBM studies in patients with PD reporting an association between cognitive impairment and GM reduction in several areas. These include the prefrontal, temporal, parietal, and occipital lobes, with the latter areas more involved in patients with PD with dementia. ${ }^{34,35}$ A more recent VBM study has reported GM cortical reduction in parietal and occipital areas in patients with PD who performed poorly on visuospatial/visuoperceptual tests. ${ }^{36}$ Moreover, previous work has shown that ventricular enlargement is associated with early cognitive impairment in patients with PD. ${ }^{37}$ Although suggested by our cognitive results and previous VBM studies, we did not find GM loss in anterior cortical areas in the whole PD population relative to HC. This apparent inconsistency might reflect the relatively early stage of disease in our PD population: The executive dysfunction detected might not yet be reflected in neuroanatomic changes detectable with VBM, which could emerge with disease progression. Nevertheless, our results are consistent with several imaging studies reporting an association between reduced cerebral glucose uptake in extensive posterior cortical areas and cognitive impairment in patients with PD. ${ }^{38-40}$

Taken together, our imaging and neuropsychological findings highlight the role of cognitive neural networks in the pathophysiology of FOG in PD. It has been demonstrated that patients with FOG have a pathologic gait pattern, ${ }^{41}$ even between FOG episodes. However, the degree of these gait alterations has not been correlated with the asymmetry in PD motor signs. ${ }^{42}$ This lack of correlation suggests that more complex networks are likely involved in their pathogenesis. As reported, previous studies have shown an association between frontal dysfunction and FOG in patients with PD. ${ }^{6,7,30}$ Recent studies ${ }^{43,44}$ have demonstrated, by evaluating the influence of space perception on gait in patients with $\mathrm{PD}$, that visuospatial ability appears to be more greatly affected in patients with PD who experience FOG. Furthermore, external visual, mental, and auditory cues (ie, extrinsic drivers) can usually improve FOG. On the basis of previous results as well as those currently presented, we speculate that a specific executive-visuospatial dysfunction may represent the cortical substrate that, in concert with the above-mentioned pathologic gait pattern, could make a subset of patients with PD prone to freeze.

In PD, automaticity is impaired; therefore, automatic movements, such as gait, depend largely on the frontal voluntary system involved in producing internally driven movements (ie, intrinsic drivers). On one hand, our study confirmed that FOG might be related to frontal networks dysfunction; on the other hand, it showed that FOG is associated with reduced GM volume in occipitoparietal pathway areas that likely underlie a dysfunction on spatial perception and discrimination, as also evidenced by neuropsychological results. Visuospatial and visuoperceptual abilities might physiologically play a key role in providing compensation through external cues when intrinsic drivers, relying on frontoexecutive networks, are impaired. Therefore, we further speculate that FOG could be due to a dysfunction of frontoexecutivebased intrinsic drivers, ${ }^{45}$ not compensated by adequate occipitoparietal-based external cues (ie, external drivers). This speculation is supported by correlation analyses demonstrating that a decreased GM volume in the left occipital cortex was associated with higher FOG-Q scores.

\section{Conclusions}

Our data provide further insight into the mechanisms underlying the complex and disabling symptom of FOG in PD. We believe that cognitive impairment, namely executivevisuospatial dysfunction, and structural GM loss in posterior cortical regions may be associated with the development of FOG. However, the present study has some limitations worth noting. First, we relied on self-report of FOG in our patients, which may be influenced by biased recall. While ideal, clinical assessment of FOG represents significant challenges because the behavior is episodic and unpredictable, often not appearing during evaluations. Second, we assessed a relatively small sample of patients, and further studies including larger populations are needed to confirm these preliminary observations.

\section{Acknowledgments}

The authors thank Antonio Cerasa for data analysis assistance and Antonella Paccone for technical assistance.

DISCLOSURES: M. Amboni-RELATED: research support from Boehringer Ingelheim, Novartis, Lundbeck. She has received salary from IDC Hermitage-Capodimonte and the Department of Neurological Sciences-University of Napoli. M. Picillo—RELATED: salary from the Department of Neurological Sciences, University of Napoli. P. Barone-RELATED: honoraria as a Consultant and Advisory Board Member for Novartis, Schwarz Pharma/UCB, Merck-Serono, Eisai, Solvay, General Electric, Bohringer Ingelheim, Teva, and Lundbeck, and he has received research support from Boehringer Ingelheim, Novartis, Schwarz Pharma/UCB, Merck-Serono, Solvay, and Lundbeck. He has received payment for lectures and for the development of educational presentations from Boehringer Ingelheim, Teva, Novartis, Lundbeck, and Merck-Serono. He has received salary from the Department of Neurological Sciences, University of Napoli.

\section{References}

1. Giladi N, Nieuwboer A. Understanding and treating freezing of gait in parkinsonism, proposed working definition, and setting the stage. Mov Disord 2008; 23(suppl 2):S423-25

2. Moore O, Peretz C, Giladi N. Freezing of gait affects quality of life of peoples with Parkinson's disease beyond its relationships with mobility and gait. Mov Disord 2007;22:2192-95

3. Giladi N. Freezing of gait. Clinical overview. Adv Neurol 2001;87:191-97

4. Bartels AL, Balash Y, Gurevich T, et al. Relationship between freezing of gait (FOG) and other features of Parkinson's: FOG is not correlated with bradykinesia. J Clin Neurosci 2003;10:584-88

5. Giladi N, McDermott MP, Fahn S, et al. Freezing of gait in PD: prospective assessment in the DATATOP cohort. Neurology 2001;56:1712-21

6. Amboni M, Cozzolino A, Longo K, et al. Freezing of gait and executive functions in patients with Parkinson's disease. Mov Disord 2008;23:395-400

7. Naismith SL, Shine JM, Lewis SJ. The specific contributions of set-shifting to freezing of gait in Parkinson's disease. Mov Disord 2010;25:1000-04

8. Shine JM, Naismith SL, Lewis SJ. The pathophysiological mechanisms underlying freezing of gait in Parkinson's Disease. J Clin Neurosci 2011;18:1154-57

9. Bartels AL, Leenders KL. Brain imaging in patients with freezing of gait. Mov Disord 2008;23(suppl 2):S461-67

10. Snijders AH, Leunissen I, Bakker M, et al. Gait-related cerebral alterations in patients with Parkinson's disease with freezing of gait. Brain 2011;134(pt 1): $59-72$

11. Schweder PM, Hansen PC, Green AL, et al. Connectivity of the pedunculopontine nucleus in parkinsonian freezing of gait. Neuroreport 2010;21:914-16

12. Gibb WR, Lees AJ. The relevance of the Lewy body to the pathogenesis of idiopathic Parkinson's disease. J Neurol Neurosurg Psychiatry 1988;51:745-52

13. Emre M, Aarsland D, Brown R, et al. Clinical diagnostic criteria for dementia associated with Parkinson's disease. Mov Disord 2007;22:1689-707, quiz 1837

14. American Psychiatric Association. Diagnostic and Statistical Manual of Mental Disorders. 4th ed. Washington, DC; American Psychiatric Association; 1994

15. Giladi N, Shabtai H, Simon ES, et al. Construction of freezing of gait questionnaire for patients with Parkinsonism. Parkinsonism Relat Disord 2000;6:165-70 16. Fahn S, Elton R. Unified Parkinson's Disease Rating Scale. In: Fahn S, Marsden 
CD, Jenner P, et al., eds. Recent Developments in Parkinson's Disease. Florham Park, New Jersey: MacMillan Health Care Information; 1987:153-163

17. Standardization and classification of Neuropsychological tests: the Italian Group on the Neuropsychological Study of Aging [in Italian]. Ital J Neurol Sci 1987;(suppl 8):1-120

18. Ashburner J, Friston KJ. Unified segmentation. Neuroimage 2005;26:839-51

19. Ashburner J. A fast diffeomorphic image registration algorithm. Neuroimage 2007;38:95-113

20. Good CD, Johnsrude IS, Ashburner J, et al. A voxel-based morphometric study of ageing in 465 normal adult human brains. Neuroimage 2001;14(1 pt 1): 21-36

21. Yogev-Seligmann G, Hausdorff JM, Giladi N. The role of executive function and attention in gait. Mov Disord 2008;23:329-42, quiz 472

22. Al-Yahya E, Dawes H, Smith L, et al. Cognitive motor interference while walking: a systematic review and meta-analysis. Neurosci Biobehav Rev 2011;35:715-28

23. Giladi N, Hausdorff JM. The role of mental function in the pathogenesis of freezing of gait in Parkinson's disease. J Neurol Sci 2006;248:173-76

24. Okuma Y, Yanagisawa N. The clinical spectrum of freezing of gait in Parkinson's disease. Mov Disord 2008;23(suppl 2):S426-30

25. Helmich RC, de Lange FP, Bloem BR, et al. Cerebral compensation during motor imagery in Parkinson's disease. Neuropsychologia 2007;45:2201-15

26. Lewis GN, Byblow WD, Walt SE. Stride length regulation in Parkinson's disease: the use of extrinsic, visual cues. Brain 2000;123(pt 10):2077-90

27. Bartels AL, de Jong BM, Giladi N, et al. Striatal dopa and glucose metabolism in PD patients with freezing of gait. Mov Disord 2006;21:1326-32

28. Lyoo CH, Aalto S, Rinne JO, et al. Different cerebral cortical areas influence the effect of subthalamic nucleus stimulation on parkinsonian motor deficits and freezing of gait. Mov Disord 2007;22:2176-82

29. Shine JM, Ward PB, Naismith SL, et al. Utilising functional MRI (fMRI) to explore the freezing phenomenon in Parkinson's disease. J Clin Neurosci 2011;18:807-10

30. Amboni M, Barone P, Picillo M, et al. A two-year follow-up study of executive dysfunctions in parkinsonian patients with freezing of gait at on-state. Mov Disord 2010;25:793-95

31. Chow TW, Cummings J. Frontal-subcortical circuits. In: Miller BL, Cummings J, eds. The Human Frontal Lobes: Functions and Disorders: (Science And Practice Of Neuropsychology Series). New York: The Guilford Press; 2007

32. Ungerleider LG, Courtney SM, Haxby JV. A neural system for human visual working memory. Proc Natl Acad Sci U S A 1998;95:883-90

33. Kravitz DJ, Saleem KS, Baker CI, et al. A new neural framework for visuospatial processing. Nat Rev Neurosci 2011;12:217-30

34. Burton EJ, McKeith IG, Burn DJ, et al. Cerebral atrophy in Parkinson's disease with and without dementia: a comparison with Alzheimer's disease, dementia with Lewy bodies and controls. Brain 2004;127(pt 4):791-800

35. Nagano-Saito A, Washimi $Y$, Arahata $Y$, et al. Cerebral atrophy and its relation to cognitive impairment in Parkinson disease. Neurology 2005;64:224-29

36. Pereira JB, Junque C, Marti MJ, et al. Neuroanatomical substrate of visuospatial and visuoperceptual impairment in Parkinson's disease. Mov Disord 2009;24:1193-99

37. Dalaker TO, Zivadinov R, Ramasamy DP, et al. Ventricular enlargement and mild cognitive impairment in early Parkinson's disease. Mov Disord 2011;26:297-301

38. Hu MT, Taylor-Robinson SD, Chaudhuri KR, et al. Cortical dysfunction in non-demented Parkinson's disease patients: a combined (31)P-MRS and (18)FDG-PET study. Brain 2000;123(pt 2):340-52

39. Hosokai Y, Nishio Y, Hirayama K, et al. Distinct patterns of regional cerebral glucose metabolism in Parkinson's disease with and without mild cognitive impairment. Mov Disord 2009;24:854-62

40. Lyoo CH, Jeong Y, Ryu YH, et al. Cerebral glucose metabolism of Parkinson's disease patients with mild cognitive impairment. Eur Neurol 2010;64:65-73

41. Hausdorff JM, Schaafsma JD, Balash Y, et al. Impaired regulation of stride variability in Parkinson's disease subjects with freezing of gait. Exp Brain Res 2003;149:187-94

42. Plotnik M, Giladi N, Balash Y, et al. Is freezing of gait in Parkinson's disease related to asymmetric motor function? Ann Neurol 2005;57:656-63

43. Almeida QJ, Lebold CA. Freezing of gait in Parkinson's disease: a perceptua cause for a motor impairment? J Neurol Neurosurg Psychiatry 2010;81:513-18

44. Cowie D, Limousin P, Peters A, et al. Insights into the neural control of locomotion from walking through doorways in Parkinson's disease. Neuropsychologia 2010;48:2750-57

45. Hallett M. The intrinsic and extrinsic aspects of freezing of gait. Mov Disord 2008;23(suppl 2):S439-40 